\title{
A PRELIMINARY EXPLORATION OF DEEP LEARNING TO IDENTIFY UNQUALIFIED USERS AND LOW QUALITY NUTRITION ADVICE ON TWITTER
}

\author{
Guto Leoni ${ }^{1}$, Patricia Endo ${ }^{1}$, Pierangelo Rosati ${ }^{2}$ and Theo Lynn ${ }^{2}$ \\ ${ }^{1}$ Centro de Informática, Universidade Federal de Pernambuco, Recife, Brazil \\ ${ }^{2}$ Irish Institute for Digital Business, DCU Business School, Dublin, Ireland
}

\begin{abstract}
The promotion of healthy nutrition and diet advice is a key tenet of public health strategies to combat obesity worldwide. Increasingly, social media is a major source of health information however is not subject to the same filtering and quality control as required by public health bodies or commercial sources. Consequently, unqualified users, poor quality nutrition and diet information, and social bots are an increasing feature of the nutrition and diet discourse on social media. Such activity may result in adverse impacts to the public and interfere with official public health education and promotion campaigns. In this paper, we propose a deep learning model based on a Long Short-Term Memory (LSTM) architecture to detect (i) unqualified Twitter accounts, and (ii) tweets featuring low quality nutritional advice. We test the proposed model on dataset comprised of tweet data and profiles associated with over $1.2 \mathrm{~m}$ tweets on nutrition and diet over a 16 -month period from January 2018 to April 2019. Preliminary results suggest that the LSTM performs significantly better for profiles than tweets, averaging between $87 \%$ and $94 \%$ for accuracy, sensitivity, precision and F1-Score, and achieving $47 \%$ for specificity. These values are much lower for the tweets classifier.
\end{abstract}

\section{KEYWORDS}

Deep Learning, Twitter, Nutrition, Diet, eHealth, Public Health Communication

\section{INTRODUCTION}

Increased consumption of energy-dense, nutrient-poor foods that are high in fat, sugar and salt, and reduced levels of physical activity are common factors attributed to increased risk of noncommunicable disease (Waxman, 2004). Increased body mass indexes can result in cardiovascular diseases, diabetes, musculo-skeletal disorders, and some cancers (WHO, 2020). Over 2.3 billion people worldwide are overweight or obese (WHO, 2020) placing a significant burden on not only health systems, but society as whole through reduced quality of life and lost economic productivity (Dee et al., 2014). The main public health strategy for combatting obesity is a combination of public health education and the promotion of healthy diets and physical activity.

Social media is a major source of nutrition and diet information for the general public and plays a significant role in inspiring food choices and food preparation (Pilgrim \& Bohnet-Joschko, 2019; Delgado et al. 2014). Despite this, the content posted on social media is not subject to the same degree of filtering and quality control by professional gatekeepers common in either public health bodies or commercial sources (Sinapuelas et al., 2019). As such, the nutrition and diet discourse on social media comprises a wide range of actors with varying qualifications and offering content of variable quality. These include traditional public health information sources such as health services, hospitals, and doctors, but also food producers, promoters and advocates of special diets and weight loss programs, celebrities, chefs, amongst others (Byrne et al., 2017; Ramachandran et al., 2018; Lynn et al., 2020). Eysenbach (2008) refers to this phenomenon as apomediation - a health information seeking strategy where the public receive "guidance" from the crowd, peers and others, using networked collaborative filtering processes, and without the same restrictions in access to information.

Unfortunately, health information on social media is prone to being out of date, incomplete, and inaccurate (Sineapuelas et al., 2019). Research suggests that nutrition and health content on social media may present opinion as fact, be shared and promoted without the support of accepted scientific evidence or is contrary to 
public policy, and in extreme cases may be deceptive, unethical and misleading (Vestergaard, 2019; Al Khaja et al., 2018; Forrest, 2019; Pilgrim \& Bohnet-Joschko, 2019; Lynn et al., 2020). As well as interfering with sound nutrition education and public health policy, reliance on poor quality nutrition information on social media can lead to the public pursuing treatments for weight loss or special diets without sufficient consideration of potential side-effects, and/or delay or failure to seek or continue legitimate medical treatment (Smith \& Jones, 2012; Ramachandran et al., 2018; Vestergaard, 2019).

Researchers have proposed a wide range of solutions for countering (i) actors who may lack suitable qualifications, have conflicts of interest, or are malicious, and (ii) reducing the impact of poor-quality content (Lynn et al., 2020). These include strategies for identifying and labelling low quality content and the accounts that disseminate messages containing such content. Assuming a pliant publisher, Lewandowsky et al. (2012) suggest that the most effective counter strategies for such messaging are (i) warnings at the time of initial exposure, (ii) repetition of retractions, if such retractions are made, and (iii) corrections that tell an alternative store that fills a coherence gaps otherwise left by the retraction. Such warnings have been implemented on popular social media networks, including Twitter and Facebook, in the case of COVID-19 and the US Presidential Elections (Wells \& Seetharaman, 2020). Similarly, research by Ozturk et al. (2015) suggests that exposing people to information that refutes unvalidated information or warns that the statement has appeared on 'rumor' websites could reduce the spread of such information. However, Ozturk et al. (2015) did not explore how such unvalidated information might be identified on scale. While there is an emerging literature base on the use of machine learning to analyze Twitter datasets in relation to nutrition and diet (see, for example, Widener \& Li, 2014; Turner-McGrievy \& Beets, 2015; Eriksson-Backa et al., 2016; Karami et al., 2018; Lynn et al., 2020), no study was identified applying deep learning for the classification of expertise or content quality in the nutrition and diet discourse on Twitter.

The objective of this paper is to explore the efficacy of deep learning for identifying (i) unqualified Twitter accounts, and (ii) nutrition advice that is not in line with the healthy eating guidelines. In this paper, we focus on the performance analysis of two long short-term memory (LSTM) artificial recurrent neural network algorithms. We constructed a binary classifier for each classification and tested them on a dataset comprising over 1.2 million English language tweets featuring the phrase 'healthy diet' or the hashtag \#healthydiet published over a 16-month period from January 2018 to April 2019, and the corresponding user profiles. By identifying a fast and scalable mechanism for detecting unqualified users and low quality nutrition and diet content, social media platforms can label such users and content appropriately.

The remainder of this paper is organized as follows. Next, we present a description of the data, the LSTM models, and the evaluation metrics used in our work. Our preliminary results are presented and discussed in Section 3. The paper concludes with a discussion of limitations and future avenues for research.

\section{DATA AND METHODS}

Twitter was selected as the empirical context of this study for a number of reasons. First, it is widely used for health surveillance and research despite not being representative of the general public (Sinnenberg et al., 2017). Second, it is actively used by a significant volume of users from a wide variety of backgrounds. Over 125 million users were active daily during 2018-19, the period under examination in this study (Twitter, 2020). Third, it is largely an open network and facilitates the connection, sharing and consumption of content between both acquaintances and strangers (Lynn et al., 2015). Fourth, the widespread use of hashtags (\#) enables users to identify other users and content, and form ad hoc communities around those hashtags (He \& Luo, 2016).

Using Twitter's enterprise API platform, GNIP, we prepared a dataset of all English language tweets featuring the phrase 'healthy diet' or the hashtag \#healthydiet from January 2018 to April 2019. This includes $1,212,318$ tweets posted by 629,608 discrete Twitter users. $45 \%$ of the tweets are original posts, $48 \%$ are retweets while the remaining $7 \%$ are replies. 7,300 out of the $629,608(1.2 \%)$ of the users in our dataset are verified (Table 1).

As discussed earlier, a number of early works have used machine learning techniques to classify nutrition and diet-related tweets. In this study, we use a Long Short-Term Memory (LSTM) artificial recurrent neural network, a form of deep learning, to construct two binary classifiers to detect (i) unqualified Twitter accounts, and (ii) tweets featuring low-quality nutritional advice. Firstly, four independent human coders, all of whom had studied nutrition and diet at third level, manually classified 888 profiles as qualified (102) or unqualified 
(786) based on their Twitter bio. Similarly, 2,200 tweets were manually classified as low quality $(1,792)$ or high quality advises (408) in comparison with the Irish healthy eating guidelines (HSE, 2020). Secondly, we processed the text content in profiles and tweets and converted each word into a representative vector using a tokenizer. Deep learning models require that all inputs must be of the same size. However, the content included in users' profiles and tweets may have different sizes. In order to overcome this issue, we applied a padding technique to make all vectors the same length.

Table 1. Dataset overview

\begin{tabular}{|c|c|c|}
\hline Message type & No. of tweets & $\%$ of tweets \\
\hline Original Tweets & 545,543 & $45 \%$ \\
\hline Retweets & 581,913 & $48 \%$ \\
\hline Replies & 84,862 & $7 \%$ \\
\hline \multirow[t]{2}{*}{ Total } & $1,212,318$ & $100 \%$ \\
\hline & No. of users & $\%$ of users \\
\hline Total & 629,608 & \\
\hline Verified & 7,300 & $1 \%$ \\
\hline
\end{tabular}

Both LSTM models consist of six layers. The first layer is an embedding layer responsible to convert the words into a vector representation. In order to avoid adjusting the parameters of this layer during the training, we used a transfer learning technique to reduce the training time. Specifically, we used a pre-trained model named fastText that was trained using Wikipedia texts composed of more than nine billion words (Mikolov et al., 2017). The following three LSTM layers include 100 units each and are designed to handle sequential data (in this case, the text from Twitter's API). For these three layers, we used tangent hyperbolic and sigmoid as the activation and recurrent activation functions respectively, and a dropout technique with probability of $20 \%$ in order to mitigate overfitting (LeCun et al., 2015).

Finally, we added two fully connected layers to define the final outcome; the first layer has 50 units while the second only has two units (in order to define the final classification). For these two final layers, we used the sigmoid activation function. The two LSTM models were trained on the manually coded data using the Adam algorithm and binary cross entropy as a loss function. We trained the LSTM models through 50 epochs, with the batch size equal to 32 . The batch size defines the numbers of samples from the training dataset that will be propagated through the network at a time in training. Finally, the models were applied to the full dataset.

\subsection{Evaluation Metrics}

Five commonly used metrics in machine and deep learning literature, namely sensitivity, specificity, accuracy, precision, and F1-Score, were used to evaluate each LSTM model. Sensitivity (or true positive rate) is the probability of a positive test result amongst those having the target condition i.e. among unqualified users or low-quality tweets as identified based on the manual coding. It is calculated as:

$$
\text { sensitivity }=\frac{T P}{T P+F N}
$$

Specificity (or true negative rate) is the probability of a negative test result amongst those without the target condition i.e. among qualified users or high-quality tweets as identified based on manual coding. It is calculated using the following equation:

$$
\text { specificity }=\frac{T N}{T N+F P}
$$


Accuracy is the rate of correct classification and is a function of sensitivity and specificity. It is calculated as:

$$
\text { accuracy }=\frac{T P+T N}{T P+F P+T N+F N}
$$

A precision score of $100 \%$ for binary classification means that every item labeled as belonging to the positive class does indeed belong to the positive class i.e. when compared to the training data, unqualified accounts were categorized as such, low quality tweets were assigned to the low quality tweets category, and so on. It is calculated as:

$$
\text { precision }=\frac{T P}{T P+F P}
$$

Finally, the F1-score is used to evaluate the overall performance of the proposed models as it is a function of both precision and sensitivity. The F1-score is particularly useful when working with imbalanced datasets such as the one used in this study as it provide a better measure of the incorrectly classified cases than the Accuracy metric. The F1-score is calculated as:

$$
F 1=2 \times \frac{\text { precision }_{\mu} \times \text { sensitivity }_{\mu}}{\text { precision }_{\mu}+\text { sensitivity }_{\mu}}
$$

\section{RESULTS AND DISCUSSION}

We carried out 30 simulations for training and testing the models. Table 2 summarizes the average and standard deviation of the evaluation metrics outlined in Section 2.1.

Table 2. LSTM Model Evaluation Metrics

\begin{tabular}{lccccc}
\hline & Accuracy & Sensitivity & Specificity & Precision & F1-score \\
\hline Profiles & $0.8720 \pm 0.0145$ & $0.9388 \pm 0.0155$ & $0.4711 \pm 0.0925$ & $0.9147 \pm 0.0131$ & $0.9263 \pm 0.0084$ \\
\hline Tweets & $0.6571 \pm 0.0259$ & $0.6540 \pm 0.0675$ & $0.6601 \pm 0.0580$ & $0.6596 \pm 0.0284$ & $0.6547 \pm 0.0353$ \\
\hline
\end{tabular}

The LSTM algorithm performs significantly better for profiles than tweets, averaging between $87 \%$ and $94 \%$ for accuracy, sensitivity, precision and F1-Score, and achieving $47 \%$ for specificity. For tweets this range is much lower, approximately $66 \%$ for all scores. As such, the simple answer is that deep learning is more effective at discerning qualified and unqualified profiles than high- and low-quality nutritional advice as measured by consistency with healthy eating guidelines.

With regards to classifying Twitter profiles, the classifier results indicate that $96 \%$ of the profiles in our dataset were unqualified, that is to say the text in their profiles did not present signals that they were suitably qualified to give nutritional and dietary advice. With regards to content, the classifier results indicate that $79 \%$ of the content was not aligned with health guidelines.

Our dataset comprises of hundreds of thousands of profiles and millions of tweets consisting of unstructured text. As such, the dataset has high dimensionality and low definition. This is particularly the case with tweets, which have greater heterogeneity than profiles. As such, more conventional classification techniques such as logistic regression and support vector machine may not be accurate or efficient. Our preliminary results suggest that the overwhelming majority of the accounts in the healthy diet discourse on Twitter are not sufficiently qualified, and the overwhelming majority of content is not consistent with public health guidelines.

Unfortunately, these preliminary findings come with their own health warning. While deep learning is very effective with dealing with high dimensionality problems, it presents interpretability challenges. For example, while results of the deep learning models examined are generally consistent with the findings presented in related studies (e.g. Lynn et al., 2020), they are inconsistent with that of the informed public. For example, although the LSTM classifier for profiles scored well in evaluations, it still classified the World Health Organization (WHO) and the UK National Health Service (NHS) incorrectly based on the text of their profile 
(see Table 3 below). We find a similar false classification in the classification of advice. Both issues may be due to the relatively small training dataset when compared to the overall dataset, or to the language and words used in the profile and tweets, and the parameters set for the binary results.

Table 3. Deep learning classification outcome - Examples

\begin{tabular}{|c|c|c|}
\hline Category & $\begin{array}{c}\text { User } \\
\text { screen name }\end{array}$ & Bio \\
\hline \multirow{4}{*}{$\begin{array}{l}\text { Accounts } \\
\text { classified } \\
\text { incorrectly }\end{array}$} & NHS & $\begin{array}{l}\text { Each week a new person curates @NHS and shares their NHS story. } \\
\text { \#HelloMyNameIs Tom, I'm a Clinical Nurse Educator working@GolborneMedical } \\
\text { and @WLondonCCG }\end{array}$ \\
\hline & WHO & $\begin{array}{l}\text { Official Twitter account of the World Health Organization, the United Nations' } \\
\text { health agency }\end{array}$ \\
\hline & wef & $\begin{array}{l}\text { The international organization for public private cooperation. Follow us for } \\
\text { research, insight and analysis on global issues }\end{array}$ \\
\hline & NINR & $\begin{array}{l}\text { Official Twitter account of NIH National Institute of Nursing Research. Following } \\
\& \text { followers do not equal endorsement. Privacy policy: http://go.usa.gov/Dz4C. }\end{array}$ \\
\hline \multirow{4}{*}{$\begin{array}{l}\text { Accounts } \\
\text { classified } \\
\text { correctly }\end{array}$} & daradietitian & $\begin{array}{l}\text { Mum of 3. Registered Dietitian. GI nutrition, Low FODMAP \& IBS, Sports } \\
\text { Nutrition, Anti-diets. Allergic to Nutribabble. MINDI, SEDI, CORU }\end{array}$ \\
\hline & judithdietitian & $\begin{array}{l}\text { I am dietitian with an interest in the diets of children and their parents. I have set up } \\
\text { my private practice to work flexibly around my own young family. }\end{array}$ \\
\hline & conorkerley & $\begin{array}{l}\text { Clinical Research Specialist \& Dietitian. MINDI. @TrueHealthINIT Council. } \\
\text { Asthmatic and PwMS. Evidence over tradition/opinion! 樂 }\end{array}$ \\
\hline & christianassad & $\begin{array}{l}\text { Preventive Interventional Cardiologist/ Director of The CardioMetabolic } \\
\text { Clinic/Interested in therapeutic applications of LCHF/iFasting/Ketogenic/RealFood } \\
\text { diets }\end{array}$ \\
\hline \multirow{4}{*}{$\begin{array}{l}\text { Advice } \\
\text { classified } \\
\text { incorrectly }\end{array}$} & GI & $\begin{array}{l}\text { Therefore it is important to not simply add olive oil or nuts to one's current diet but } \\
\text { to adopt the [Mediterranean diet] plan in its entirety }\end{array}$ \\
\hline & AGE & $\begin{array}{l}\text { Mediterranean diet nutrients tied with healthy brain aging } \\
\text { https://t.co/2Y1Vc7BPCP }\end{array}$ \\
\hline & FAO & $\begin{array}{l}\text { A healthy diet starts with safe food. We need to ensure availability and access to } \\
\text { safe food now and in the future. } \mathrm{http}: / / \mathrm{bit} . l \mathrm{ly} / 2 \mathrm{VFqtF} 2\end{array}$ \\
\hline & NHS & $\begin{array}{l}\text { So to help with increased appetite and missing the hand to mouth action of } \\
\text { smoking, rather than going for the crisps and biscuits try a bag of carrot sticks, } \\
\text { celery and mage tout (my daily snack pack for work days - you get used to it } \\
\text { \#behaviourchange) }\end{array}$ \\
\hline \multirow{4}{*}{$\begin{array}{l}\text { Advice } \\
\text { classified } \\
\text { correctly }\end{array}$} & healthguide911 & $\begin{array}{l}\text { \#Fruit \#juice is a far less \#healthy option than a real piece of fruit } \\
\text { https://t.co/jE2pMCwJoh \#healthydiet }\end{array}$ \\
\hline & GoMediiOfficial & $\begin{array}{l}\text { Eating a diet rich in some vegetables and fruits as part of an overall healthy diet } \\
\text { may protect against certain types of cancers. \#cancer \#wellness } \\
\text { https://t.co/0BvEI5Qup2 }\end{array}$ \\
\hline & DrCopperAfrica & $\begin{array}{l}\text { Start your week by being healthy. Fruits and vegetables are an essential part of a } \\
\text { balanced diet along with keeping properly hydrated. \#DrinkWaterBetter with an } \\
\text { original @DrCopperAfrica bottle. \#Copper4Life }\end{array}$ \\
\hline & Go_Go_Beauty & $\begin{array}{l}\text { Eat a healthy diet! A healthy diet can help you look and feel your best. Eat plenty } \\
\text { of fruits, vegetables, whole grains and lean proteins. }\end{array}$ \\
\hline
\end{tabular}

Our deep learning model had difficulty distinguishing legitimate public health organizations primarily because of the general way that they described themselves. In this study, highly qualified nutrition and diet profiles need to make explicit references to specific qualifications. In the same way, it may be difficult for the general public to relate to a large monolithic brand such as the WHO, World Economic Forum (WEF), and the NHS, whose operations are so wide that they either do not associate them with nutrition and diet or the content feed is not targeted enough for individual users. Such organizations need to consider whether it is more prudent to develop segment-specific accounts focused on nutrition or even sub-topics, where they can build and interact with a specific audience more specifically.

With regards to messaging, the false negatives often feature words not typically associated with good nutrition and diet e.g. smoking, crisps and biscuits. In other cases, the message is inconclusive. While humans can derive the meaning from these tweets, it represents a difficult task for machines. It is possible that this can 
be resolved using larger training datasets and using different language model pre-training techniques such as bidirectional encoder representations from transformers (BERT) (Devlin et al., 2018). Such technologies are used by Google for search engine ranking and may offer substantial performance improvements from both a technical and communications perspective. In addition, a form of ensemble model could be developed to take into account both the source credibility, in this case the Twitter account, and the quality of the content i.e. the Tweet.

\section{CONCLUSION}

The consequences to the public following poor nutritional dietary advice can result in variety of adverse effects including malnutrition and, in extreme cases, death. While the promotion of healthy diets is a key building block of public health education, such messaging is lost in a deluge of content, much of which is based on opinion and unsubstantiated by scientific evidence. With COVID-19, social networking platforms have demonstrated that, where sufficiently motivated, they are willing to take action to mitigate the dissemination of health misinformation including removal of content and labelling content with warnings. Unfortunately, unlike COVID-19, scientific and policy perspectives on nutrition and diet are less binary and often are inflected by a particular worldview. Furthermore, publishers of nutrition and diet advice represent a wide range of actors and qualifications, and the language used in advice and messaging is much more ambiguous than in COVID-19. As such, the identification of "unqualified" advisor and "poor quality" content on social media is not only much more difficult but represents a greater challenge to freedom of speech, particularly as imminent harm to the public may be less evident.

Recent research suggests significant volumes of both misinformation and unsubstantiated opinion, as well as an increasing prevalence of content polluters, including spam and bots in the nutrition and diet discourse (Vestergaard, 2019; Al Khaja et al., 2018; Forrest, 2019; Pilgrim \& Bohnet-Joschko, 2019; Lynn et al., 2020). Against this backdrop, this preliminary study makes an important contribution. It presents one of the first applications of deep learning, and LSTM specifically, for classifying social media users and social media content in the nutrition and diet domain. In doing so, it identifies challenges in operationalizing LSTM for use on social media platforms while also identifying opportunities and challenges for researchers wishing to use such technologies and analytical techniques in research studies. Furthermore, it highlights some practical considerations for public health communicators with regards to not only writing content for humans but for future machine-based moderation including search engines using technologies such as BERT for ranking. Finally, it raises questions for social networking platforms with regards to their responsibilities regarding moderating such nutrition and diet content, and policymakers regarding the balance between the right of free speech and public health concerns, and the role policymakers play in regulating social media platforms as conduits of health information.

This is a preliminary study and, as such, is not without limitations. The dataset, while substantial, represents subset of the wider discourse on nutrition and diet discussion on Twitter and is limited to one language, English. As discussed earlier, while the models performed well against the dataset, there were a number of misclassifications. Future work will compare the performance of different machine learning and deep learning techniques, ensembles, and make use of a larger training dataset. In addition, the social media data exhaust includes a wide range of additional signals (e.g., verification status) that may also increase accuracy. Finally, rather than a binary outcome, future work will examine a ranking system or scale that may provide more nuanced and accurate results.

\section{ACKNOWLEDGEMENT}

This paper was partly funded by Safefood, the all-island implementation body set up under the British-Irish Agreement to promote awareness and knowledge of food safety and nutrition issues on the island of Ireland. 


\section{REFERENCES}

Al Khaja, K.A., AlKhaja, A.K. and Sequeira, R.P., 2018. Drug information, misinformation, and disinformation on social media: a content analysis study. Journal of public health policy, Vol. 39, No. 3, pp.343-357.

Ayoob, K.T., Duyff, R.L. and Quagliani, D., 2002. Position of the American Dietetic Association: food and nutrition misinformation. Journal of the American Dietetic Association, Vol. 102, No. 2, pp.260-266.

Byrne, E., Kearney, J. and MacEvilly, C., 2017. The role of influencer marketing and social influencers in public health. Proceedings of the Nutrition Society Irish Section Conference 2017, Belfast, United Kingdom, 76(OCE3).

Dee, A., Kearns, K., O’Neill, C., Sharp, L., Staines, A., O’Dwyer, V., Fitzgerald, S. and Perry, I.J., 2014. The direct and indirect costs of both overweight and obesity: a systematic review. BMC research notes, Vol. 7, No. 1, pp.1-9.

Delgado, J., Johnsmeyer, B. and Balanovsky, S., 2014. Millennials eat up YouTube food videos. Available at: https://www.thinkwithgoogle.com/marketing-strategies/video/millennials-eat-up-youtube-food-videos/ (last accessed on 5 December 2020).

Devlin, J., Chang, M.W., Lee, K. and Toutanova, K., 2018. Bert: Pre-training of deep bidirectional transformers for language understanding. arXiv preprint arXiv:1810.04805.

Eriksson-Backa, K., Holmberg, K. and Ek, S., 2016. Communicating diabetes and diets on Twitter-a semantic content analysis. International Journal of Networking and Virtual Organisations, Vol. 16, No. 1, pp.8-24.

Eysenbach, G., 2008. Medicine 2.0: social networking, collaboration, participation, apomediation, and openness. Journal of medical Internet research, Vol. 10, No. 3, pp. 1-11.

Forrest, A., 2019. "Social media influencers give bad diet and fitness advice eight times out of nine, research reveals". Independent, 30 April. https://www.independent.co.uk/news/health/social-media-weight-loss-diet-twitterinfluencersbloggers-glasgow-university-a8891971.html

He, L. and Luo, J., 2016, December. "What makes a pro eating disorder hashtag": Using hashtags to identify pro eating disorder tumblr posts and Twitter users. In 2016 IEEE International Conference on Big Data (Big Data), Washington D.C., United States, pp. 3977-3979.

HSE, 2020. Healthy Eating Guidelines. Available at: https://www.hse.ie/eng/about/who/healthwellbeing/our-priorityprogrammes/heal/healthy-eating-guidelines/ (last accessed on 5 December 2020).

Karami, A., Dahl, A.A., Turner-McGrievy, G., Kharrazi, H. and Shaw Jr, G., 2018. Characterizing diabetes, diet, exercise, and obesity comments on Twitter. International Journal of Information Management, Vol. 38, No. 1, pp.1-6.

LeCun, Y., Bengio, Y., \& Hinton, G. (2015). Deep learning. Nature, Vol. 521, No. 7553, pp. 436-444.

Lewandowsky, S., Ecker, U. K., Seifert, C. M., Schwarz, N., \& Cook, J. (2012). Misinformation and its correction: Continued influence and successful debiasing. Psychological science in the public interest, Vol. 13, No. 3, 106-131.

Lynn, T., Healy, P., Kilroy, S., Hunt, G., Van Der Werff, L., Venkatagiri, S. and Morrison, J., 2015, July. Towards a general research framework for social media research using big data. In 2015 IEEE International Professional Communication Conference (IPCC), Limerick, Ireland, pp. 1-8

Lynn, T., Rosati, P., Leoni Santos, G. and Endo, P.T., 2020. Sorting the Healthy Diet Signal from the Social Media Expert Noise: Preliminary Evidence from the Healthy Diet Discourse on Twitter. International Journal of Environmental Research and Public Health, Vol. 17, No. 22, pp. 1-28.

Mikolov, T., Grave, E., Bojanowski, P., Puhrsch, C., \& Joulin, A. (2017). Advances in pre-training distributed word representations. arXiv preprint arXiv:1712.09405.

Ozturk, P., Li, H., \& Sakamoto, Y. (2015, January). Combating rumor spread on social media: The effectiveness of refutation and warning. In 2015 48th Hawaii International Conference on System Sciences, Hawaii, United States, pp. 2406-2414.

Pilgrim, K. and Bohnet-Joschko, S., 2019. Selling health and happiness how influencers communicate on Instagram about dieting and exercise: Mixed methods research. BMC public health, Vol. 19, No. 1, pp. 1-9.

Ramachandran, D., Kite, J., Vassallo, A.J., Chau, J.Y., Partridge, S., Freeman, B. and Gill, T., 2018. Food trends and popular nutrition advice online-implications for public health. Online Journal of Public Health Informatics, Vol. 10, No. 2, pp.1-15.

Sinapuelas, I.C. and Ho, F.N., 2019. Information exchange in social networks for health care. Journal of Consumer Marketing, Vol. 36, No. 5, pp. 692-702.

Sinnenberg, L., Buttenheim, A.M., Padrez, K., Mancheno, C., Ungar, L. and Merchant, R.M., 2017. Twitter as a tool for health research: a systematic review. American journal of public health, Vol. 107, No. 1, pp. e1-e8.

Smith, A. and Jones, G., 2012. Miracle pills and fireproof trainers: user endorsement in social media. Bmj, Vol. 345, pp. 1-3.

Turner-McGrievy, G.M. and Beets, M.W., 2015. Tweet for health: using an online social network to examine temporal trends in weight loss-related posts. Translational behavioral medicine, Vol. 5, No. 2, pp.160-166. 
Twitter, 2020. Selected company metrics and financials. Available at: https://investor.twitterinc.com/home/default.aspx (last accessed on 5 December 2020).

Vestergaard, M.G. and Nielsen, L.M., 2019. The Danish veterinary and food administration's fight against fake nutrition news on digital media. Tidsskrift for Medier, Erkendelse Og Formidling, Vol. 7, No. 2, pp.21-21.

Waxman, A., 2004. WHO global strategy on diet, physical activity and health. Food and nutrition bulletin, Vol. 25, No. 3, pp.292-302.

Wells, G. and Seetharaman, D., 2020. Facebook, Twitter Grapple With Trump's False Election Claims. Wall Street Journal, 4 November. Available at: https:/www.wsj.com/articles/facebook-twitter-grapple-with-trumps-false-election-claims11604532938 (last accessed on 5 December 2020).

Widener, M.J. and Li, W., 2014. Using geolocated Twitter data to monitor the prevalence of healthy and unhealthy food references across the US. Applied Geography, Vol. 54, pp.189-197.

WHO, 2020. Obesity. Available at: https://www.who.int/health-topics/obesity\#tab=tab_1 (last accessed on 5 December 2020). 